\title{
Criminologie
}

\section{Les prisons du Québec ; ghetto des pauvres}

\section{André Campeau}

Volume 9, numéro 1-2, 1976

L’emprisonnement au Québec

URI : https://id.erudit.org/iderudit/017051ar

DOI : https://doi.org/10.7202/017051ar

Aller au sommaire du numéro

Éditeur(s)

Les Presses de l'Université de Montréal

ISSN

0316-0041 (imprimé)

1492-1367 (numérique)

Découvrir la revue

Citer cet article

Campeau, A. (1976). Les prisons du Québec ; ghetto des pauvres. Criminologie, 9(1-2), 60-86. https://doi.org/10.7202/017051ar d'utilisation que vous pouvez consulter en ligne.

https://apropos.erudit.org/fr/usagers/politique-dutilisation/ 
LES PRISONS DU QUÉBEC; GHETTO DES PAUVRES

La recherche ${ }^{1}$ dont nous présentons la synthèse dans cet article consistait en une analyse du profil des personnes emprisonnées pour défaut de paiement d'amende durant l'année 1974, pour le territoire relevant du centre de détention de Québec (prison d'Orsainville), et une analyse des sentences selon les types de délits. Parallèlement, nous nous sommes efforcés d'établir des comparaisons avec les résultats obtenus à la suite de précédentes recherches effectuées à la prison de Bordeaux (centre de détention de Montréal). En effet, au mois de juin 1974, Claude Menghile et Ronald Pépin effectuaient une étude portant sur le centre de détention de Montréal. Intitulée «Description de la population de l'établissement de détention de Montréaln, cette étude faite sous la direction du professeur Pierre Landreville, constituait une vue d'ensemble de la population carcérale de cette prison provinciale, entre le $1^{\text {er }}$ octobre 1972 et le $1^{\text {er }}$ octobre 1973. Ả l'aide de données statistiques et de tableaux, les auteurs ont tracé le profil des détenus présents durant cette période et celui de ceux présents le 9 octobre 1973. La recherche de Menghile et Pépin fut complétée par celle de $\mathbf{M}^{11}$ Michele Hudon concernant l'ensemble de la population du centre de détention de Montréal pour l'année 1973. Bien que la période étudiée n'était pas exactement la même, les similitudes de résultats obtenus sont significatives. En effet, les deux recherches forment un tout qui donne une idée très précise de la population carcérale de Bordeaux, et en même temps un aperçu fort intéressant de la population carcérale des établissements de détention du Québec.

1. Étude sur les «Modalités et les conséquences de l'emprisonnement à défaut de paiement d'amende," recherche dirigée par Alice Parizeau, CICC, Université de Montréal, mars 1975. 
C'est pourquoi les études dirigées par Pierre Landreville et celles de $\mathbf{M}^{\text {tle }}$ Hudon ont été utilisées comme base de comparaison pour la recherche faite à la prison d'Orsainville.

Selon Menghile et Pépin, $47,7 \%$ des gens qui ont été emprisonnés, durant la période étudiée, l'ont été pour défaut de paiement d'amende. Cela signifie que si toutes les personnes auxquelles une amende a été imposée avaient acquitté celle-ci, la population du centre de détention de Montréal aurait diminué de près de la moitié. Les pourcentages obtenus par Menghile et Pépin, en ce qui a trait à l'emprisonnement à défaut de paiement d'amende, ont été mis en doute et on a fortement critiqué la méthode employée qui était celle de l'échantillonage. 11 était d'autant plus essentiel, par conséquent, de vérifier si les résultats obtenus par Menghile et Pépin pouvaient être confirmés dans le cadre d'une étude sur une autre prison du Québec, et sur la base de relevés compilés pour l'année suivante, soit 1974.

La cueillette des données concernant les détenus de la prison d'Orsainville a été faite à partir des copies des livres d'écrou se trouvant aux bureaux de la Direction générale de la probation et des établissements de détention du Québec. C'est à cet endroit que sont conservés, en effet, tous les renseignements relatifs à l'ensemble de la population carcérale de chacun des établissements de détention du Québec. Contrairement aux études faites précédemment, portant sur le centre de détention de Montréal, nous avons recueilli les renseignements sur toutes les personnes détenues à la prison d'Orsainville au cours de 1974 pour défaut de paiement d'amende. Nos résultats ne sont donc pas basés sur un échantillon, mais bien sur l'ensemble des détenus de cette catégorie, ce qui leur confère le maximum de précision.

La méthode de travail consistait à prendre tous les livres d'écrou relatifs au centre de détention de Québec et de consulter une par une les feuilles d'ècrou, correspondant chacune à une admission. Ensuite, toutes les personnes qui ont été admises à titre de prévenu ont été éliminées, pour ne retenir que les détenus qui ont été incarcérés pour défaut de paiement d'amende. De ces trois groupes, prévenus, détenus "ordinaires", détenus pour défaut de paiement d'amende, seuls les détenus de cette troisième catégorie ont fait l'objet de notre enquête en ce qui a trait à leur profession, le degré de scolarité, l'âge et l'état civil, ainsi que la nature du délit pour lequel ils ont été condamnés, la Cour devant laquelle 
ils ont été entendus et la sentence qui leur a été imposée en termes d'amende et, à défaut, du nombre de jours de prison. Par ailleurs, nous avons relevé également les dates d'admission et de libération de l'institution carcérale et de la justification de cette libération. Par "justification de la libération", nous entendons la raison pour laquelle la personne a été libérée, soit parce que sa peine était purgée dans son entier ou parce qu'elle a pu acquitter l'amende, alors même qu'elle était déjà incarcérée.

Dans le premier temps, nous avons donc essayé de tracer le profil socio-économique des personnes détenues pour défaut de paiement d'amende et dans un deuxième temps, d'analyser le contenu des sentences à option.

Les renseignements relatifs à l'âge ont été classés selon les mêmes groupes qu'ont utilisés Menghile et Pépin, soit 18-25, 26-35, $36-45,46-55$ et 66 ans et plus. En plus de nous permettre de comparer nos résultats avec ceux obtenus dans cette étude, cette classification offre l'avantage de correspondre à des mini-générations. De même, les délits ont été classés selon les mêmes divisions qui ont servi dans les deux études précédentes.

En ce qui a trait aux sentences, la population carcérale emprisonnée pour défaut de paiement d'amende se recrute parmi les condamnés par la Cour des sessions de la paix et les Cours municipales, dont les modes de procéder semblent sensiblement différents.

Lorsqu'une personne est condamnée à l'amende devant la Cour des sessions de la paix, on n'exige pas, en pratique, que celle-ci paie immédiatement, le montant de l'amende. Le dossier est remis au greffe de la Cour qui, à partir de cet instant, prend l'affaire en charge. On écrit à la personne condamnée à l'amende pour l'aviser, même si elle est déjà au courant, qu'elle doit payer son amende avant la fin du délai fixé, délai d'au moins un mois à compter de la date du jugement. Si la personne ne peut payer dans le délai imparti, elle peut se présenter au greffe de la Cour et solliciter un nouveau délai, qui est généralement accordé à condition qu'on puisse constater qu'il s'agit là d'un retard justifiable. Lorsque tous les délais sont écoulés, un agent de la paix se présente chez la personne concernée avec l'obligation de la conduire en prison, si elle ne peut verser le montant de l'amende sur-le-champ.

Lorsqu'une personne est condamnée à l'amende devant une Cour municipale, dès le prononcé de la sentence, la personne 
déclarée coupable peut être tenue de payer. Si elle ne peut le faire, elle est conduite au centre de détention pour purger le nombre de jours correspondant à ceux indiqués dans la sentence pour défaut de paiement d'amende. On évite ainsi des procédures administratives, mais la philosophie même qui a présidé à l'établissement des peines pécuniaires est faussée puisque la possibilité de choix entre l'amende et la perte de liberté devient en fait très réduite, sinon inexistante.

D'une façon générale, en outre, lorsqu'une personne est emprisonnée à défaut de paiement d'amende, elle peut être libérée avant que sa peine ne soit entièrement purgée à condition de payer l'amende qui lui a été imposée. Si cette personne a été condamnée en vertu du Code criminel, ou de certaines lois fédérales, le montant de l'amende qu'elle devra débourser est proportionnel au nombre de jours qu'il lui reste à purger par rapport à celui qui a été fixé par la sentence. Toutefois, si un individu est emprisonné parce qu'il n'a pas pu payer l'amende imposée pour une violation à une loi provinciale ou au Code municipal, il devra débourser le montant global de l'amende, peu importe le nombre de jours qu'il a déjà purgés.

Cela constitue une autre différence fondamentale au niveau du «rachat» de la liberté surtout lorsque le montant de l'amende est relativement élevé.

À la lumière de ces quelques remarques préliminaires, il est loisible de constater que le principe de l'égalité de tous les citoyens devant la loi, risque d'être souvent faussé dans les faits. La procédure de l'exécution des sentences relatives à l'amende, en tant qu'une alternative à l'emprisonnement, pénalise incontestablement les classes défavorisées.

À cet égard, par ailleurs, l'étude du profil socio-économique des prisonniers demeure, on ne peut plus révélatrice.

Ajoutons également que sur le plan des coûts supportés par les contribuables, l'économie des frais de greffe de la Cour est minime quand on la compare aux frais de la détention qui sont, il va sans dire, largement supérieurs.

C'est ainsi que la prison d'Orsainville a accueilli, au cours de l'année 1974, 2457 personnes qui ont séjourné durant une période plus ou moins longue dans cette institution. Parmi ces 2457 personnes, on retrouvait 1103 détenus et 1354 prévenus. Sur ces 
1103 détenus, on dénombrait 599 personnes incarcérées pour défaut de paiement d'amende, soit $54,3 \%$ de tous les détenus ayant séjourné à la prison d'Orsainville au cours de l'année 1974. Si toutes les personnes à qui une amende a été imposée au cours de 1974 avaient acquitté celle-ci, la population du centre de détention de Québec aurait diminué de plus de la moitié.

\section{A. LES CARACTÉRISTIQUES SOCIO-ÉCONOMIQUES DES DÉTENUS}

Les personnes détenues pour défaut de paiement d'amende sont relativement jeunes. Ce sont les moins de 25 ans qui sont les plus nombreux. Ils forment à eux seuls plus de $31.9 \%$ de tous ces détenus.

TABLEAU I

Répartition des sujets suivant l'âge

(Orsainville-1974)

\begin{tabular}{crrr}
\hline Âge & Nombre & $\%$ & \% cumulatif \\
\hline $18-25$ & 181 & 31,9 & \\
$26-35$ & 147 & 25,8 & 31,9 \\
$36-45$ & 109 & 19.2 & 57,7 \\
$46-55$ & 100 & 17.6 & 76,9 \\
$56-65$ & 24 & 4,3 & 94,5 \\
$66-+$ & 7 & 1.2 & 98,8 \\
& & & 100.0 \\
\hline
\end{tabular}

Nous remarquons que plus les détenus sont âgés, moins ils sont nombreux. La baisse la plus considérable se situe chez les gens entre 56 et 65 ans qui sont quatre fois moins nombreux que le groupe d'âge précédent. La population de la prison d'Orsainville incarcérée pour défaut de paiement d'amende se caractérise donc, en tout premier lieu, par son âge peu élevé; $57,7 \%$ de ces détenus ont moins de 35 ans, $76 \%$ moins de 45 ans.

TABLEAU 2

Répartition des sujets selon l'état civil (Orsainville-1974)

\begin{tabular}{lrr}
\hline Célibataire & 389 & $68.5 \%$ \\
Marié & 106 & $18.7 \%$ \\
Séparé & 44 & $7.7 \%$ \\
Divorcé & 23 & $4.0 \%$ \\
Veuf & $\frac{6}{568}$ & $1.1 \%$ \\
Total & & \\
\hline
\end{tabular}


Ce sont les célibataires qui forment la très grande majorité des détenus pour défaut de paiement d'amende. En effet, 68,5\% des détenus emprisonnés pour défaut de paiement d'amende sont célibataires alors que seulement $18,7 \%$ sont mariés et que $11,7 \%$ sont séparés ou divorcés.

TABLEAU 3

Répartition des sujets selon la scolarité (Orsainville-1974)

\begin{tabular}{cccc}
\hline $\begin{array}{c}\text { Degré de } \\
\text { scolarité }\end{array}$ & Nombre & $\%$ & $\begin{array}{c}\% \\
\text { cumulatif }\end{array}$ \\
\hline Nil & 5 & 0.9 & 0.9 \\
1 & 1 & 0.2 & 1.1 \\
2 & 6 & 1.1 & 2.2 \\
3 & 15 & 2,6 & 4.8 \\
4 & 26 & 4,6 & 9.3 \\
5 & 38 & 6.7 & 16.0 \\
6 & 38 & 6.7 & 22,7 \\
7 & 76 & 13.4 & 36,1 \\
8 & 64 & 11,3 & 47,4 \\
9 & 104 & 18,3 & 65.7 \\
10 & 69 & 12.1 & 77,8 \\
11 & 51 & 9.0 & 86,8 \\
12 & 37 & 6.5 & 93.3 \\
13 & 8 & 1,4 & 94.7 \\
14 & 14 & 2,5 & 97,2 \\
$15+$ & 16 & 2.8 & 100.0 \\
\hline
\end{tabular}

Les personnes détenues pour défaut de paiement d'amende ont un niveau de scolarité assez bas, comme l'indique le tableau 3 où l'on constate que $65,7 \%$ n'ont pas dépassé la neuvième année d'études et que $93,3 \%$ n'ont pas dépassé la douzième année. Si l'on considère que la population des détenus emprisonnés pour défaut de paiement d'amende se situe à $31,9 \%$ entre 18-25 ans, donc la génération ayant bénéficié de la gratuité scolaire au secondaire et en partie au CEGEP, on est surpris de constater que leur scolarité soit si réduite.

La moyenne de scolarité chez les détenus, pour défaut de paiement d'amende se situe entre la $7^{\circ}$ et la $10^{\circ}$ année, où se concentre $55,1 \%$ du total. Nous sommes donc en présence de gens ayant un niveau secondaire d'instruction, mais dont le cours n'a pas été terminé. La $9^{\circ}$ année de scolarité a été complétée par 104 détenus, ce qui représente $18,3 \%$ de l'ensemble. La $9^{\circ}$ année a toujours semblé être un point tournant chez les jeunes, qui abandonnent souvent leurs études après cette année-là; ceci correspond en fait à l'âge où ils peuvent essayer de pénétrer sur le marché 
du travail et au niveau de scolarité qui était autrefois exigé pour la plupart des métiers.

Il y a uniquement $5,3 \%$ des prisonniers qui ont un degré de scolarité plus élevé que le niveau secondaire. Parmi ces détenus plus instruits, seulement 16 , soit $2,8 \%$ du total de la population étudiée, ont un niveau de scolarité supérieure à 15 années, donc, selon toute probabilité, une formation professionnelle ou universitaire.

D’une manière générale, en outre, les personnes qui sont détenues pour défaut de paiement d'amende, se recrutent parmi une main-d'œuvre non spécialisée.

TABLEAU 4

Répartition des sujets selon loccupation (Orsainville-1974)

\begin{tabular}{lrrr}
\hline Occupation & Nombre & $\%$ & cumulatif \\
\hline Aucune (chômeurs) & 48 & 8.5 & 8.5 \\
Invalides & 7 & 1.2 & 9.7 \\
Retraités & 9 & 1.6 & 11.3 \\
Étudiants & 14 & 2.5 & 13.8 \\
Journaliers & 265 & 46.7 & 60.5 \\
Métiers divers & 214 & 37.6 & 98.1 \\
Professionnels & 11 & 1.9 & 100.0 \\
\hline
\end{tabular}

Le tableau des occupations, examiné en regard avec les résultats obtenus en ce qui concerne le niveau de scolarité des détenus, confirme l'hypothèse selon laquelle les personnes emprisonnées à défaut de paiement d'amende sont socio-économiquement désavantagées.

On constate, en effet, que $11,3 \%$ ont déclaré n'avoir aucune occupation et être chômeurs, invalides, ou retraités. Si l'on ajoute à ces individus ceux qui se sont déclarés étudiants, on arrive à la conclusion que près de $14 \%$ des personnes concernées n'avaient aucun revenu "personnel" stable avant leur condamnation. Le manque de ressources apparaît dès lors comme le facteur principal de non-paiement de l'amende.

Autre constatation qui découle directement de nos résultats sur le degré de scolarité chez les détenus, $46,7 \%$ des individus se sont déclarés journaliers. Ceci est le reflet du manque d'instruction et démontre que $46,7 \%$ des personnes avaient des revenus inférieurs à la moyenne, ou peut-être même en deçà du seuil de pauvreté, 
et pouvaient ne pas disposer de moyens nécessaires pour acquitter l'amende sur-le-champ.

On peut présumer que les personnes pratiquant divers métiers ont un revenu supérieur à celles qui sont dites «journaliers", mais il n'en demeure pas moins probable qu'elles n'étaient pas capables de verser la totalité de la somme dans un délai trop court et que le total de l'amende n'aurait pas dû être exigé sans une prise en considération de la situation réelle des concernés. Il est loisible, en somme d'avancer l'hypothèse que près de $75 \%$ de toutes les personnes emprisonnées à défaut de paiement d'amende ont un revenu qui ne leur permet pas, la plupart du temps, de pouvoir acquitter une amende sans s'endetter; cette incapacité pouvant être encore plus marquée chez les $46,7 \%$ qui se sont déclarés journaliers.

En ce qui a trait aux "professionnels» (architecte, dessinateur industriel et technicien spécialisé) ils ne forment que $1,9 \%$ du total, ce qui est légèrement inférieur au pourcentage de détenus ayant une formation académique de 15 ans et plus. Il demeure néanmoins surprenant de constater que des personnes susceptibles d'avoir un revenu supérieur à la moyenne n'aient pu acquitter l'amende. L'explication de ce phénomène réside peut-être dans le fait que la moitié de ces personnes ont comparu devant une Cour municipale et que n'ayant pas eu de délai pour payer, elles ont été incarcérées. Quant à l'autre moitié, on peut présumer que les hommes concernés étaient en fait sans emploi, mais ce sont là des hypothèses difficiles à vérifier.

\section{B. LES VARIABLES DU MONTANT DES AMENDES IMPOSÉES}

Le tableau 5, comprend les données relatives aux amendes pour lesquelles des individus ont purgé une sentence en prison au lieu de les acquitter.

Plus de la moitié des personnes emprisonnées en 1974, pour défaut de paiement d'amende, l'ont été pour des amendes inférieures à $\$ 50$, tandis que $50,7 \%$ de toutes les personnes incarcérées pour cette raison, l'ont été pour des amendes variant de 0 à $\$ 49$. De même, $68,0 \%$ de tous les individus emprisonnés pour défaut de paiement d'amende l'ont été parce qu'ils n'ont pas acquitté des amendes inférieures à $\$ 100$. Il est intéressant également de noter qu'il y a eu plus d'amendes imposées entre $\$ 100$ et $\$ 200$ qu'entre 
TABLEAU 5

Répartition des sujets selon les amendes

(Orsainville-1974)

\begin{tabular}{cccc}
\hline $\begin{array}{c}\text { Montant } \\
\text { d'amende } \\
\$\end{array}$ & $\begin{array}{c}\text { Nombre des } \\
\text { emprisonnements }\end{array}$ & $\%$ & \% cumulatif \\
\hline $0-49$ & 288 & 50.7 & 50,7 \\
$50-99$ & 98 & 17.3 & 68,0 \\
$100-199$ & 105 & 18,6 & 86,6 \\
$200-299$ & 37 & 6,7 & 93,3 \\
$300-399$ & 21 & 3,4 & 96,7 \\
$400-499$ & 6 & 1.0 & 97,7 \\
$500-999$ & 11 & 1.9 & 99,6 \\
$1000-+$ & 2 & .4 & 100,00 \\
\hline
\end{tabular}

$\$ 50$ et $\$ 100$. On remarque finalement qu'au-dessus de $\$ 200$ le nombre d'emprisonnements baisse considérablement à mesure que le montant de l'amende augmente.

It convient, par ailleurs, d'insister tout particulièrement sur le problème des emprisonnements pour non paiement d'amendes inférieures à $\$ 50$ et ce, pour diverses raisons. Comme ceux-ci concernent plus de $50 \%$ de toute cette catégorie de détenus et que les amendes de moins de $\$ 50$, plus que toutes autres, sont susceptibles d'être payées en raison de leur modicité relative, il est important de vérifier la répartition exacte des amendes inférieures à ce montant.

TABLEAU 6

Répartition des amendes de moins de $\$ 50$ (Orsainville-1974)

\begin{tabular}{lcrc}
\hline $\begin{array}{c}\text { Montant } \\
\text { d'amende } \\
\$\end{array}$ & $\begin{array}{c}\text { Nombre des } \\
\text { emprisonnements }\end{array}$ & $\%$ & \% cumulatif \\
\hline $10-18$ & 12 & 2.1 & 2,1 \\
19 & 78 & 13.7 & 15,8 \\
$20-33$ & 73 & 12.9 & 28.7 \\
34 & 56 & 9.9 & 38.6 \\
$35-48$ & 35 & 6.1 & 44,7 \\
49 & 34 & 6.0 & 50.7 \\
\hline
\end{tabular}

Le tableau 6, qui représente les amendes inférieures à $\$ 50$ a été réalisé en isolant les trois taux d'amendes les plus fréquents, soit $\$ 19, \$ 34$ et $\$ 49$.

On constate que $2,1 \%$ des personnes emprisonnées à défaut de paiement d'amende l'ont été pour des sommes telles que $\$ 18$ et moins. Ce pourcentage monte rapidement à $15,8 \%$ si on ajoute 
un dollar. Les amendes de $\$ 19$ ont été le plus souvent à l'origine d'emprisonnements $(13,7 \%)$, suivies des amendes de $\$ 34(9,9 \%)$ et celles de $\$ 49(6,0 \%)$. L'analyse de ces données, démontre, par ailleurs, qu'il existe une certaine uniformité dans l'imposition des sentences d'amendes. Comme nous l'indiquons plus loin, il s'agit principalement des causes entendues par les Cours municipales dans les cas de désordre, d'ivresse ou de flânage.

Ainsi, $15,8 \%$ des individus emprisonnés pour défaut de paiement d'amende l'ont donc été pour des sommes aussi faibles que $\$ 19$ et moins, $28,7 \%$ pour moins de $\$ 34,38,6 \%$ pour moins de $\$ 35$ (à noter l'importance des amendes de $\$ 34$ ), $44,7 \%$ pour moins de $\$ 49$ et finalement $50,7 \%$ pour moins de $\$ 50$ (à noter l'importance des amendes de \$49).

Il ressort clairement de tous ces chiffres que les hypothèses, que nous avions émises précédemment sur la situation économique des individus emprisonnés à défaut de paiement d'amende, s'avèrent exactes. En effet, malgré le niveau relativement faible de 50,7\% des amendes, les condamnés ont séjourné en prison, ce qui semble être la conséquence de manque de ressources financières. Nous voilà donc en présence d'une catégorie défavorisée de la population; celle des économiquement faibles.

\section{LES VARIABLES DE LA DURÉE D'EMPRISONNEMENT POUR DÉFAUT DE PAIEMENT D'AMENDE}

Les tableaux qui suivent ont trait aux peines de prison imposées pour le non-paiement des amendes. Néanmoins, une distinction est faite entre la peine de prison telle que fixée par la sentence et celle réellement purgée par le condamné. En effet, comme il a été expliqué précédemment, un individu qui ne verse pas l'amende doit purger le nombre de jours de prison indiqués dans la sentence, mais s'il paie avant l'expiration de sa peine, il est libéré. Il s'ensuit donc des disparités entre la peine d'emprisonnement, telle que fixée par la sentence et celle réellement purgée.

Le tableau 7 indique les peines d'emprisonnement infligées aux personnes qui n'ont pu acquitter leurs amendes. Premièrement, il y a lieu de constater que $63,4 \%$ des personnes incarcérées l'ont été pour des périodes de moins de 15 jours. Ceci semble logique étant donné que $50,7 \%$ des personnes devaient des amendes de moins de $\$ 50$. Pour leur part, les sentences d'emprisonnement de 
TABLEAU 7

Répartition des peines d'incarcération selon les sentences (Orsainville - 1974)

\begin{tabular}{lrrc}
\hline Durée & Nombre & \multicolumn{1}{c}{$\%$} & $\%$ cumulatif \\
\hline $1-15$ jours & 360 & 63.4 & 63,4 \\
$15-30$ jours & 115 & 20,2 & 83.6 \\
$31-59$ jours & 27 & 4.8 & 88.4 \\
$60-89$ jours & 47 & 8,3 & 96,7 \\
$90-119$ jours & 15 & 2,6 & 99.3 \\
4 à 6 mois & 2 & .4 & 99.6 \\
+6 mois & 2 & .4 & 100.0 \\
\hline
\end{tabular}

moins d'un mois ont constitué $83,6 \%$ de toutes les incarcérations, tandis que les sentences supérieures à un mois ne composent que $16,4 \%$ de toutes les peines imposées.

La question qui se pose est celle de savoir combien de personnes ont acquitté l'amende et ont pu sortir avant que leur peine ne soit totalement purgée.

TABLEAU 8

Répartition des sujets selon la libération

(Orsainville - 1974)

\begin{tabular}{lcc}
\hline Amende payée & 252 & $44.4 \%$ \\
$\begin{array}{l}\text { Peine purgée } \\
\text { Libération }\end{array}$ & 315 & $55,5 \%$ \\
conditionnelle & 1 & $0,2 \%$ \\
\hline
\end{tabular}

On constate d'après le tableau 8 que plus de gens ont purgé leur peine d'emprisonnement en entier $(55,5 \%)$ que de gens qui ont été capables de payer l'amende afin de racheter leur liberté $(44,4 \%)$. Comment expliquer qu'ils aient attendu d'être derrière les barreaux pour verser le montant d'argent prévu par la sentence? Doit-on conclure que le processus administratif ne tient pas compte de la capacité de payer des condamnés, ou que l'effet intimidant de l'incarcération entraîne l'intervention de la famille, ou des amis du condamné?

En effet le tableau 9 indique tout d'abord que 98 individus, soit $17,3 \%$ de toute la population emprisonnée à défaut de paiement d'amende, n'ont purgé qu'une seule journée à Orsainville. Près d'un cinquième de la population concernée a pu en somme acquitter l'amende le jour même de l'incarcération. On considère, en effet, qu'une personne qui entre dans une prison purge 1 journée, même 
TABLEAU 9

Répartition des sujers selon la longueur de la peine purgée (Orsainville - 1974)

\begin{tabular}{ccrc}
\hline Nombre de jours & Nombre de sujets & $\%$ & \% cumulatif \\
\hline 1 & 98 & 17.3 & 17.3 \\
2 & 63 & 11.1 & 28.3 \\
3 & 28 & 4.9 & 33.3 \\
4 & 18 & 3.2 & 36.4 \\
5 & 20 & 3.5 & 40.0 \\
6 & 16 & 2.8 & 42,8 \\
7 & 23 & 4.0 & 46.8 \\
8 & 93 & 16.4 & 63.2 \\
9 & 7 & 1.2 & 64.4 \\
10 & 7 & 1.2 & 65.7 \\
11 & 11 & 1.9 & 67.6 \\
12 & 24 & 4.2 & 71,8 \\
13 & 4 & 0.7 & 72,5 \\
14 & 47 & 8.3 & 82,2 \\
15 à 29 & 60 & 6.6 & 92.8 \\
30 a 59 & 34 & 1.2 & 100.8 \\
60 et & 7 & & \\
& & & \\
\hline
\end{tabular}

si elle n'y passe pas la nuit. La question qui se pose consiste à chercher la raison pour laquelle ces personnes n'ont pas acquitté l'amende avant cela. Plusieurs hypothèses sont possibles à cet égard. En ce qui a trait aux condamnés par une Cour municipale, ils ont pu ne pas disposer d'argent nécessaire faute de délai et par la suite, un membre de leur famille a pu venir l'apporter. En ce qui concerne, par contre, les condamnés par la Cour des sessions de la paix, ils ont pu être incapables, malgré les délais, de recueillir l'argent nécessaire et ce n'est que placés face à l'emprisonnement qu'ils ont pu, ou voulu, demander l'aide d'un parent, ou ami.

Les mêmes questions se posent également si l'on considère que $28,3 \%$ des individus ont acquitté leur amende après 2 jours d'incarcération. Ce sont des personnes qui ont passé la nuit en prison et dont l'amende a été acquittée le lendemain du jour où elle a été imposée. Ces gens sont dans une situation assez similaire à ceux dont l'amende a été acquittée le jour même, à la seule différence, que dans leur cas, la réaction des proches a pu être moins rapide.

Le tableau 9 indique ensuite que $16,4 \%$ des individus ont été libérés après avoir purgé 8 jours à Orsainville. Ce pourcentage élevé de libérations au bout de 8 jours s'explique en partie par le nombre élevé de personnes qui ont eu des sentences de cette durée. Par conséquent, on constate que $63,2 \%$ des individus ont 
purgé une sentence de 8 jours ou moins, ce qui est nettement supérieur au nombre de sentences prévoyant une telle période.

Finalement, il s'avère que 47 individus $(8,3 \%)$ ont purgé une peine de 14 jours, ce qui porte le nombre de peines purgées, durant moins de 15 jours, à $82,2 \%$. On constate donc un double phénomène : les individus emprisonnés à défaut de paiement d'amende purgent des périodes nettement inférieures à celles prévues par leur sentence et plusieurs d'entre eux paient leur amende très vite après leur incarcération. Ce phénomène est-il lié aux disparités des modes de procéder des Cours de justice?

\section{LES DISPARITÉS DES SENTENCES}

Les personnes détenues pour défaut d'amende sont condamnées, en effet, soit par la Cour des sessions de la paix soit par les Cours municipales. La situation de l'emprisonnement à défaut de paiement d'amende diffère de l'une à l'autre de ces cours, principalement en raison des catégories de délits et du type particulier d'accusés que l'on retrouve devant chacune d'elles.

TABLEAU 10

Répartition des condamnés selon les Cours (1974)

\begin{tabular}{lcc}
\hline Cours & Nombre & $\%$ \\
\hline $\begin{array}{l}\text { Cours municipales } \\
\begin{array}{l}\text { Cour des sessions } \\
\text { de la paix }\end{array}\end{array}$ & 337 & 59.3 \\
\hline
\end{tabular}

Près de $60 \%$ des gens qui ont été emprisonnés à défaut de paiement d'amende en 1974, provenaient des Cours municipales, alors qu'à peine $40 \%$ venaient de la Cour des sessions de la paix. Si l'on considère que cela représente environ $35 \%$ de toutes les personnes détenues à Orsainville en 1974, on constate l'importance des sentences prononcées par les juges des Cours municipales en ce qui a trait aux peines de détention. Les infractions traitées par les Cours municipales sont généralement des infractions de moindre importance, il n'en reste pas moins que $60 \%$ des personnes emprisonnées à défaut de paiement d'amende ont été condamnées par ces tribunaux. En ce qui a trait aux catégories de ces délits, elles sont indiquées au tableau 11. 
TABLEAU 1]

Répartition des sujets selon les délits (Orsainville 1974)

\begin{tabular}{|c|c|c|c|}
\hline Type de délit & Nombre & $\%$ & $\begin{array}{l}\text { \% par } \\
\text { catégorie }\end{array}$ \\
\hline \multicolumn{3}{|l|}{ Biens sans violence } & 3,5 \\
\hline Vol & 15 & 2.6 & \\
\hline $\begin{array}{l}\text { Dommages } \\
\text { Crimes contre la personne }\end{array}$ & 5 & 9 & \\
\hline \multicolumn{3}{|l|}{ Crimes contre la personne } & 0,9 \\
\hline $\begin{array}{l}\text { Voies de fait } \\
\text { Voies de fait et }\end{array}$ & 3 & .5 & \\
\hline $\begin{array}{l}\text { Voies de fait et } \\
\text { lésions corporelles }\end{array}$ & 1 & 2 & \\
\hline Rapt corporel & 1 & 2 & \\
\hline Mours avec victime (sexe) & & & 1,6 \\
\hline Attentat à la pudeur & 1 & 2 & \\
\hline Actions indécentes & 4 & .7 & \\
\hline Grossière indécence & 4 &, 7 & \\
\hline Nuisance publique sans victime & & & 42,7 \\
\hline Ivresse & 106 & 18,7 & \\
\hline Flânage & 19 & 3.3 & \\
\hline Mendicité & 8 & 1.4 & \\
\hline Désordre & 97 & 17.0 & \\
\hline Flânage et désordre & 1 & .2 & \\
\hline Ivresse et mendicité & 2 & .4 & \\
\hline Ivresse et désordre & 3 & .5 & \\
\hline Divers & 6 & 1.2 & \\
\hline \multicolumn{4}{|l|}{$\begin{array}{l}\text { Infractions relatives aux } \\
\text { véhicules moteurs }\end{array}$} \\
\hline $\begin{array}{l}\text { véhicules moteurs } \\
\text { Code de la route }\end{array}$ & 132 & 23.2 & 47,0 \\
\hline $\begin{array}{l}\text { Reglement municipal de } \\
\text { circulation }\end{array}$ & & & \\
\hline $\begin{array}{l}\text { circulation } \\
\text { Délit de fuite }\end{array}$ & 86. & 15.1 & \\
\hline Délit de fuite & 2 & .4 & \\
\hline Conduite avec facultés affaiblies & 28 & 4.9 & \\
\hline \multirow{7}{*}{$\begin{array}{l}\text { Infractions relatives à des lois } \\
\text { statutaires } \\
\text { Loi Commission des alcools } \\
\text { du Québec } \\
\text { Loi Commission d'assurance- } \\
\text { chômage } \\
\text { Loi sur stupéfiants et drogues } \\
\text { Loi de l'impôt } \\
\text { Infractions diverses }\end{array}$} & 18 & 3,4 & \\
\hline & & & 7 \\
\hline & & & 2,1 \\
\hline & 3 & .5 & \\
\hline & 4 & .7 & \\
\hline & 3 & .5 & \\
\hline & 2 & .4 & \\
\hline \multirow[t]{2}{*}{ Infractions diverses } & 14 & & 2,2 \\
\hline & $\overline{568}$ & & 100 \\
\hline
\end{tabular}

Un autre facteur, que celui de la typologie des délits, qui peut justifier l'importance du rôle des Cours municipales dans les emprisonnements à défaut de paiement d'amende, est celui relatif aux délais accordés par les juges pour acquitter l'amende. Autant on peut justifier ainsi qu'un nombre plus important de gens est emprisonné, autant il serait loisible de s'attendre à ce qu'une plus 
grande proportion d'individus paie son amende après une ou deux journées de détention.

TABLEAU 12

Tableau des libérations selon les Cours (1974)

\begin{tabular}{lccccc}
\hline Cours & Amende payée & & Peine purgée & Total \\
\hline $\begin{array}{l}\text { Municipale } \\
\begin{array}{l}\text { Sessions de } \\
\text { la paix }\end{array}\end{array}$ & $151 \quad(44,8 \%)$ & 186 & $(55,2 \%)$ & 337 \\
\hline
\end{tabular}

Or, le tableau 12 démontre très clairement qu'il n'y a aucune différence entre la Cour des sessions de la paix et les Cours municipales en ce qui a trait aux libérations. La question des délais de détention jouerait donc un rôle relativement négatif, sinon on trouverait un pourcentage plus élevé d'amendes payées chez les individus condamnés par les Cours municipales. Cette constatation n'est en fait que le reflet de la situation économique des personnes en question, qui seraient incapables de réunir suffisamment d'argent pour acquitter leur amende. Les concernés semblent être dans un état d'infériorité économique où la moindre épargne est impossible et où la nécessité d'acquitter une amende présente un défi quasi insurmontable. La question du délai de paiement pourrait par contre jouer un rôle plus important si les personnes concernées étaient dans une meilleure situation financière. Quoi qu'il en soit, il semble qu'un très grand nombre d'emprisonnements à défaut de paiement d'amende pourrait être évité si les personnes déclarées coupables disposaient, dans tous les cas, d'un délai plus long pour payer leur amende.

En dehors du problème des délais, il y a certainement d'autres facteurs pouvant expliquer le fait que $60 \%$ des personnes détenues pour défaut de paiement d'amende proviennent des Cours municipales. Il est possible, par exemple, que les amendes y soient plus élevées tandis que les peines d'emprisonnement tellement courtes qu'il est considéré comme plus "payant" de purger la peine d'incarcération. On peut se demander aussi si des différences socio-économiques significatives n'existent pas entre la majorité des personnes provenant des Cours municipales et celles provenant de la Cour des sessions de la paix. 
TABLEAU 13

Répartition des peines d'emprisonnement selon les Cours (1974)

\begin{tabular}{rrrrrr}
\hline Jours & Cour municipale (\%) & Cour des sessions (\%) & Total \\
\hline $0-14$ & 158 & $(46,8 \%)$ & 102 & $(44,1 \%)$ & 260 \\
$15-30$ & 116 & $(34,4 \%)$ & 99 & $(42,4 \%)$ & 215 \\
$31-59$ & 15 & $(4,5 \%)$ & 12 & $(5,9 \%)$ & 27 \\
$60-89$ & 37 & $(11,0 \%)$ & 10 & $(4,2 \%)$ & 47 \\
$90-119$ & 9 & $(2,7 \%)$ & 6 & $(2,6 \%)$ & 15 \\
$120-149$ & 2 & $(0.6 \%)$ & 0 & $(0,0 \%)$ & 2 \\
$150+$ & 0 & $(0,0 \%)$ & 2 & $(0,8 \%)$ & $\frac{2}{568}$ \\
& 337 & $(100 \%)$ & 213 & $(100 \%)$ & \\
\hline
\end{tabular}

Les peines d'emprisonnement pour défaut de paiement d'amende imposées par les Cours municipales ne semblent en aucune façon inférieures à celles imposées par la Cour des sessions de la paix. Alors que les sentences inférieures à 15 jours sont sensiblement comparables $(46,8 \%-44,1 \%)$, on constate une moins forte proportion de sentences d'un mois et moins au niveau des Cours municipales qu'à celui de la Cour des sessions de la paix. Alors que $86,5 \%$ des personnes traduites devant la Cour des sessions de la paix ont obtenu des sentences inférieures à un mois, seulement $81,2 \%$ de celles traduites devant les Cours municipales ont reçu des sentences similaires. Cette différence de $5 \%$, qui peut sembler minime au départ, prend de l'importance lorsqu'on considère que les infractions municipales sont beaucoup moins graves que celles entendues devant les Cours de sessions de la paix.

De plus, $15,5 \%$ des sentences imposées par les Cours municipales étaient d'une durée d'un à trois mois, alors que seulement $10,1 \%$ des sentences de la Cour des sessions étaient limitées à cette même période de temps. On peut donc conclure à une sévérité relativement plus grande des Cours municipales, sévérité qui ne favorise certes pas une fréquentation accrue de nos établissements de détention par les personnes condamnées à l'amende par ces Cours. Il faut donc chercher ailleurs l'explication du $60 \%$ que constitue le groupe d'individus condamnés par les Cours municipales.

Le tableau 14 indique très clairement que les amendes imposées par les Cours municipales sont nettement inférieures à celles imposées par la Cour des sessions de la paix. On constate tout d'abord que $73,2 \%$ des amendes imposées par les Cours 
TABLEAU 14

Répartition des amendes selon les cours (1974)

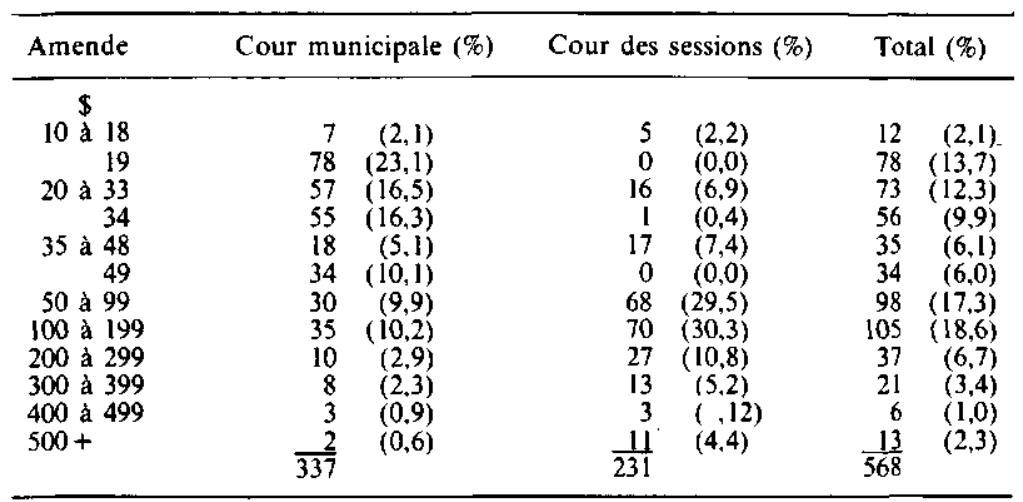

municipales sont inférieures à $\$ 50$ alors que seulement $16,9 \%$ de celles imposées par la Cour des sessions de la paix le sont. De même, alors que $82,1 \%$ des amendes des Cours municipales sont inférieures à $\$ 100$ et $92,3 \%$ à $\$ 200$, seulement $46,4 \%$ de celles imposées par la Cour des sessions de la paix sont moindres que $\$ 100$, et $76 \%$ moindres que $\$ 200$. On dénote donc que $70 \%$ des amendes imposées par la Cour des sessions de la paix se situent entre $\$ 50$ et $\$ 300$.

Cette différence très importante qui existe entre le niveau des amendes imposées par les Cours municipales et celui des amendes imposées par la Cour des sessions de la paix, s'explique en fait par la nature même des causes qu'elles entendent respectivement. La Cour des sessions de la paix est appelée à imposer des amendes pour des délits plus importants, relevant par exemple du Code criminel, alors que les Cours municipales ont à statuer à la suite de commission des délits qu'on ne peut qualifier de majeurs.

Il n'en reste pas moins que la lecture des tableaux 13 et 14 indique clairement que les Cours municipales imposent des amendes plus faibles et des peines alternatives d'incarcération d'une durée aussi longue, sinon plus que pour la Cour des sessions de la paix. Le ratio qui existe entre les amendes et la peine d'emprisonnement prévue par la sentence serait donc beaucoup plus basse pour la Cour des sessions de la paix que pour les Cours municipales. En d'autres termes, les Cours municipales imposeraient beaucoup plus de jours d'emprisonnement par dollar d'amende que la Cour des sessions de la paix. 
On ne peut conclure, en somme, que les personnes condamnées à l'amende par les Cours municipales choisissent de préférence l'emprisonnement parce que cela est "plus avantageux" pour elles économiquement parlant. La question du délai jouerait donc un rôle important en tant que moyen d'éviter des emprisonnements inutiles, mais, comme il a été indiqué plus haut, la pénible situation économique de la plupart des concernés annihile pratiquement l'effet positif d'un délai, qui ne s'accompagne pas des versements de l'amende répartis sur la même période de temps, payable progressivement par exemple.

Le tableau 14 indique également que les taux des amendes varient beaucoup d'une Cour à l'autre. C'est ainsi, par exemple, que les amendes de $\$ 19, \$ 34$ et de $\$ 49$, très souvent imposées par les Cours municipales, ne le sont pratiquement jamais par les Cours des sessions de la paix. On peut donc conclure, à partir de là, qu'il existe un plus grand souci d'uniformisation des peines pécuniaires au niveau des Cours municipales que des Cours des sessions de la paix.

TABLEAU 15

Répartition des peines purgées selon les cours (1974)

\begin{tabular}{ccccc}
\hline Nombre de jours & Cour municipale & \multicolumn{2}{c}{$\begin{array}{c}\text { Cour des sessions } \\
\text { de la paix }\end{array}$} & Total \\
\hline 1 & $59(17,5 \%)$ & $39(16,9 \%)$ & 98 \\
2 & $32(9,5 \%)$ & $31(13,4 \%)$ & 63 \\
3 & $13(3,9 \%)$ & $15(6,5 \%)$ & 28 \\
4 & $10(3,0 \%)$ & $8(3,5 \%)$ & 18 \\
5 & $12(3,6 \%)$ & $8(3,5 \%)$ & 20 \\
6 & $9(2,7 \%)$ & $7(3,0 \%)$ & 16 \\
7 & $13(3,9 \%)$ & $10(4,3 \%)$ & 23 \\
8 & $61(18,1 \%)$ & $32(13,9 \%)$ & 93 \\
$9-14$ & $56(17,0 \%)$ & $44(17,3 \%)$ & 100 \\
$15-30$ & $35(10,6 \%)$ & $25(10,7 \%)$ & 60 \\
$31-59$ & $27(8,0 \%)$ & $7(3,0 \%)$ & 34 \\
$60-89$ & $4(1,0 \%)$ & $2(0,7 \%)$ & 6 \\
$90+$ & $0(0,0 \%)$ & $1(0,4 \%)$ & 1 \\
\hline
\end{tabular}

D'après le tableau $15,80,4 \%$ des personnes incarcérées à Orsainville pour défaut de paiement d'amende, à la suite d'un jugement prononcé par la Cour municipale, ont purgé moins de 15 jours et le pourcentage est de $85,2 \%$ soit relativement comparable, pour les personnes condamnées par les Cours des sessions de la paix. Si l'on tient compte du fait que la Cour des sessions a prononcé 
plus de sentences inférieures à 15 jours, on peut dire grosso modo qu'il n'y a pas de différence entre la Cour des sessions et les Cours municipales au niveau de la longueur des peines réellement purgées. Nous constatons également qu'environ la même proportion de gens ont acquitté leur amende dès le premier jour, ce qui laisse croire cependant que si les personnes traduites devant la Cour municipale avaient joui d'un délai, le nombre de 59 aurait diminué sensiblement. Il ne faut pas considérer en tous les cas l'équivalence des pourcentages au niveau de la première journée et croire par là qu'un délai n'aurait pas entraîné de différence, mais bien espérer qu'une très grande partie de ces 59 personnes aurait pu éviter l'emprisonnement si elles avaient eu un délai pour payer.

La même constatation pourrait également s'appliquer à ceux qui ont acquitté l'amende dès la deuxième journée de leur emprisonnement, pour les raisons qui ont été exposées au préalable. On peut donc affirmer que malgré que la durée de l'incarcération réelle est la même tant pour les condamnés par la Cour des sessions de la paix que pour ceux des Cours municipales, il n'en reste pas moins que comme les amendes sont nettement inférieures à la Cour municipale, des délais accordés pour les acquitter pourraient jouer un rôle efficace de dissuasion dans le sens de diminution du nombre d'emprisonnements à défaut de paiements d'amende.

Par ailleurs, les délais de paiement ne sont pas le seul facteur - qui puisse expliquer que $60 \%$ des personnes emprisonnées à défaut

TABLEAU 16

Répartition des principaux délits selon les cours (1974)

\begin{tabular}{|c|c|c|c|c|}
\hline Cour municipale & Infraction & Nombre & $\%$ & $\%$ cumulatif \\
\hline & $\begin{array}{l}\text { Ivresse } \\
\text { Désordre } \\
\text { Flanâge } \\
\text { Circulation }\end{array}$ & $\begin{array}{r}106 \\
94 \\
19 \\
86\end{array}$ & $\begin{array}{r}31.2 \\
27.9 \\
5.6 \\
25.5\end{array}$ & $\begin{array}{l}31.2 \\
59.1 \\
64.7 \\
90.2\end{array}$ \\
\hline \multirow[t]{2}{*}{$\begin{array}{l}\text { Cour des Sessions } \\
\text { de la paix }\end{array}$} & Infraction & Nombre & $\%$ & $\%$ cumulatif \\
\hline & $\begin{array}{l}\text { Code de la route } \\
\text { Autres infractions re- } \\
\text { latives aux véhicules } \\
\text { moteurs }\end{array}$ & 132 & 52.8 & 52,8 \\
\hline
\end{tabular}


de paiement d'amende, proviennent des Cours municipales. Au contraire, il semble bien que le véritable problème découle plutôt de la nature même des causes que doivent entendre ces Cours et de la situation des personnes qui en sont déclarées coupables.

Les infractions que la Cour des sessions de la paix doit le plus souvent entendre sont des infractions relatives à la conduite des véhicules moteurs. En effet, 73,6\% des emprisonnements à défaut de paiement d'amende qui originaient des décisions de la Cour des sessions de la paix étaient reliés à la conduite des véhicules moteurs. Les infractions reliées aux véhicules moteurs sont des infractions pour lesquelles la récidive est pratiquement inexistante, c'est-à-dire qu'il est très rare que la même personne soit emprisonnée à deux reprises pour le non-paiement de l'amende. Généralement, une première expérience semble suffisante et lorsque la personne se retrouve devant la même alternative, elle choisit, sans hésiter, de payer l'amende. On ne peut donc pas parler de «délinquants d'habitude" lorsque l'on réfère à des personnes déclarées coupables d'infractions relatives aux véhicules moteurs.

La même constatation s'applique également aux individus ayant été déclarés coupables de violation d'un règlement municipal de la circulation. Ceux-ci forment $25,5 \%$ de tous les emprisonnements à défaut de paiement d'amende provenant des Cours municipales. À l'opposé, la situation est différente pour les personnes déclarées coupables de nuisance publique.

En effet, près de $65 \%$ de toutes les infractions entendues par les Cours municipales et pour lesquelles il y a eu un emprisonnement pour défaut de paiement d'amende, étaient des infractions de nuisance publique. Or, c'est dans le domaine des infractions de nuisance publique que l'on retrouve le plus haut taux de récidive.

TABLEAU 17

Répartition des délits de nuisance publique selon l'âge (1974)

\begin{tabular}{lcrrrrr}
\hline Áge & Désordre & Ivresse & Flânage & Total & $\%$ & \% cumulatif \\
\hline $18-25$ & 19 & 5 & 11 & 35 & 15,9 & 15,9 \\
$26-35$ & 13 & 10 & 4 & 27 & 12,3 & 28,2 \\
$36-45$ & 18 & 29 & 1 & 48 & 22,0 & 50,2 \\
$46-55$ & 35 & 36 & 3 & 74 & 33,8 & 84,0 \\
$56-65$ & 8 & 20 & - & 28 & 12,4 & 96,4 \\
$66+$ & $\frac{6}{94}$ & $\frac{6}{106}$ & $\overline{19}$ & $\frac{7}{219}$ & 3,6 & 100,0 \\
& $\frac{1}{94}$ & & & & & \\
\hline
\end{tabular}


On est en présence, en effet, d'une catégorie d'individus que l'on peut qualifier de "vagabonds" et de "robineux" qui viennent en moyenne de 3 à 5 fois par année à Orsainville, et toujours pour les mêmes infractions pour des périodes variant entre 8 à 30 jours, selon le nombre d'incarcérations antérieures.

Le tableau 17 indique les âges des personnes qui ont été emprisonnées pour défaut de paiement d'amende après avoir été déclarées coupables de nuisance publique. Nous remarquons tout d'abord que l'âge des personnes emprisonnées à la suite d'un délit de nuisance publique est nettement supérieur à la moyenne des âges de toutes les personnes emprisonnées pour défaut de paiement d'amende.

Alors que $31,9 \%$ des personnes emprisonnées à défaut de paiement d'amende avaient moins de 25 ans, seulement $15,9 \%$ des personnes trouvées coupables de nuisance publique avaient moins de 25 ans. De même, alors que dans toute la population incarcérée pour non paiement d'amende, $57,7 \%$ des individus avaient moins de 35 ans, seulement $28,2 \%$ des emprisonnements à la suite de délits de nuisance publique concernaient des individus de moins de 35 ans. Nous retrouvons donc une catégorie nettement plus âgée d'individus pour les infractions de nuisance publique.

Le tableau 17 indique que près de $50 \%$ des personnes déclarées coupables de nuisance publique et emprisonnées parce qu'elles n'ont pu acquitter l'amende, étaient âgées de plus de 45 ans.

Comme nous l'avons déjà mentionné nous avons considéré chaque emprisonnement individuellement, sans relation avec les autres emprisonnements qui seraient purgés par la même personne.

TABLEAU 18

Répartition des délits de nuisance publique selon la récidive (1974)

Nombre d'emprisonnements par individu

Nombre d'individus

$\begin{array}{lr}1 & 62 \\ 2 & 16 \\ 3 & 10 \\ 4 & 9 \\ 5 & 4 \\ 6 & 3 \\ 7 & 3\end{array}$


Afin de vérifier l'état de la récidive chez les prisonniers trouvés coupables de nuisance publique en 1974, nous avons étudié, cependant chaque dossier individuellement, afin de relever le nombre d'emprisonnements pour défaut de paiement d'amende d'une même personne.

\section{E. LES CARACTÉRISTIQUES DES RÉCIDIVISTES}

En 1974, le nombre d'emprisonnements à défaut de paiement d'amende, pour nuisance publique, était de 219 , mais ne concernait en fait que 107 individus trouvés coupables de ce genre de délits. Ainsi que l'indique le tableau 18, seulement 62 individus ont été déclarés coupables et condamnés à l'emprisonnement pour défaut de paiement d'amende pour une seule infraction de nuisance publique. Ces 62 personnes correspondent à 62 emprisonnements, chacune d'elles n'étant venue qu'une seule fois à Orsainville en 1974. Fait intéressant à noter, la très grande majorité de ces 62 personnes est plus jeune que la moyenne des personnes ayant été déclarées coupables de nuisance publique.

Pour l'ensemble de ce groupe 45 individus ont purgé 157 emprisonnements à défaut de paiement d'amende pour nuisance publique, ce qui représente $71,6 \%$ des infractions de nuisance publique, $47,5 \%$ de ceux provenant des Cours municipales et $27,6 \%$ de tous les emprisonnements pour défaut de paiement d'amende pour 1974. Cela veut donc dire que si l'on avait traité de façon différente ces 45 individus, la population carcérale, détenue pour défaut de paiement d'amende, aurait diminuée de plus du quart! Ce sont ces indivitus, en outre, qui ont purgé leur peine en totalité et qui augmentent donc la moyenne de la durée de l'incarcération réelle ordonnée par les Cours municipales.

\section{F. ASPECTS TECHNIQUES DE L'EMPRISONNEMENT $\AA$ DÉFAUT DE PAIEMENT D'AMENDE}

Lorsqu'une personne est déclarée coupable d'une infraction pour laquelle le juge peut prononcer une sentence à option, soit l'imposition de l'amende, et, à défaut de paiement, l'emprisonnement, le sort immédiat du prévenu est entre les mains du magistrat. En effet, quand un juge prononce une sentence à option, il jouit d'une très large discrétion et d'une part d'appréciation dans le choix de la mesure à appliquer. Cette discrétion joue à deux niveaux : 
celui du choix du montant de l'amende et celui du choix du nombre de jours que devra purger le coupable s'il ne paye pas sur-le-champ, ou dans un délai accordé à cette fin.

La discrétion du juge lorsqu'il impose une amende est limitée par la loi, celle-ci lui laisse cependant une très grande liberté d'appréciation. C'est ainsi, par exemple, que la loi québécoise des infractions sommaires "limite" l'action du juge à moins de $\$ 500$ et à défaut de paiement, à 6 mois d'emprisonnement au maximum, ce qui lui confère une très grande latitude. On peut donc affirmer que la discrétion du juge demeure la règle en la matière.

Le deuxième volet de la discrétion du juge, en matière d'imposition de sentence à option, a trait aux jours d'emprisonnement, qu'il doit imposer, pour défaut de paiement d'amende. C'est finalement cet aspect de la question qui nous touche de plus près. Quand le juge impose son amende, il doit y faire correspondre un certain nombre de journées d'emprisonnement en cas de défaut de paiement de celle-ci. Or, une fois de plus, il trouve sa seule limite dans la loi qui lui indique un maximum, mais plus rarement un minimum. Il choisit donc seul, à sa discrétion, le nombre de jours d'emprisonnement approprié.

L'analyse des tableaux statistiques suivants indiquent les équivalences entre les montants des amendes et le nombre de jours d'incarcération, ainsi que leurs variables selon les Cours.

TABLEAU 19

Équivalences entre le montant de l'amende et le nombre de jours de prison prévus par la. sentence (Cour des sessions de la paix) (1974)

\begin{tabular}{|c|c|c|c|c|c|c|c|c|c|}
\hline $\begin{array}{l}\text { Jours de } \\
\text { prison }\end{array}$ & $1-15$ & $16-30$ & $31-45$ & $\begin{array}{r}\text { Mon } \\
45.60\end{array}$ & $\begin{array}{r}\tan t \text { de } \\
61-100\end{array}$ & $\begin{array}{l}\text { lamende } \\
|0|-200\end{array}$ & $\stackrel{\$}{201-500}$ & $500+$ & Total \\
\hline $1 \mathrm{à} 8 \mathrm{j}$. & 5 & 11 & 15 & 10 & 24 & 18 & 6 & - & 89 \\
\hline 9 à $15 \mathrm{j}$. & - & - & - & 13 & 11 & 2 & 9 & 1 & 57 \\
\hline 16 à $30 \mathrm{j}$ & - & 4 & 1 & 1 & 6 & 28 & 15 & - & 55 \\
\hline 31 à $60 \mathrm{j}$. & - & - & - & - & - & 5 & 7 & 6 & 18 \\
\hline 61 à $90 \mathrm{j}$. & - & - & - & - & - & 3 & 5 & 2 & 10 \\
\hline $91+$ & - & - & - & - & - & - & - & 2 & 2 \\
\hline Total & 5 & 15 & 16 & 24 & -41 & 77 & 42 & 11 & 231 \\
\hline
\end{tabular}

Sur la base du tableau 19 on constate en premier lieu que la diversité des sentences est très grande, et en deuxième lieu que les amendes sont relativement élevées par rapport aux jours de détention prévus. Toutefois, avant d'examiner plus en détail le 
tableau 19 , nous reproduisons celui qui indique les correspondances pour la Cour municipale, afin de pouvoir analyser ensuite les deux parallèlement.

TABLEAU 20

Équivalences entre le montant de l'amende et le nombre de jours de prison prévus par la sentence (Cours municipales) (1974)

\begin{tabular}{|c|c|c|c|c|c|c|c|c|c|}
\hline $\begin{array}{l}\text { Jours de } \\
\text { prison }\end{array}$ & $1-15$ & $16-30$ & $31-45$ & $\begin{array}{c}\text { Mont } \\
46-60\end{array}$ & $\begin{array}{c}\operatorname{tant} d e \\
61-100\end{array}$ & $\begin{array}{c}\text { amende } \mathrm{e} \\
101-200\end{array}$ & $201-500+$ & & Total \\
\hline 1 à $8 \mathrm{j}$ & 2 & 113 & 18 & 7 & 6 & 2 & - & - & 148 \\
\hline 9 à $15 \mathrm{j}$ & - & 21 & 24 & 1 & 6 & 15 & - & - & 67 \\
\hline 16 a $30 \mathrm{j}$ & - & l & 31 & 7 & 2 & 8 & 6 & - & 55 \\
\hline 31 à $60 \mathrm{j}$ & - & - & - & 32 & 2 & 4 & 16 & 1 & 55 \\
\hline 61 à $90 \mathrm{j}$ & - & - & - & - & - & 9 & - & l & 10 \\
\hline $91+$ & - & - & - & - & 2 & - & - & - & 2 \\
\hline Total & 2 & 135 & 73 & 47 & 18 & 38 & 22 & 2 & 337 \\
\hline
\end{tabular}

On remarque tout d'abord, sur la base du tableau 20, que malgré que les amendes soient nettement moindres pour les condamnés des Cours municipales, on retrouve des peines d'emprisonnement sensiblement comparables. Cela signifie que, dollar pour dollar, un individu purge moins de jours d'emprisonnement, pour défaut de paiement d'amende, quand il est condamné par la Cour des sessions de la paix que par une Cour municipale. C'est ainsi qu'on peut présumer, sur la base des données disponibles, qu'un individu qui a reçu entre $\$ 31$ et $\$ 45$ d'amende devant la Cour municipale, risque d'être condamné pour une période allant de 16 à 30 jours d'emprisonnement, tandis que s'il avait reçu une telle amende devant la Cour des sessions de la paix, il est fort probable qu'il n'aurait eu qu'entre 1 et 8 jours d'emprisonnement.

En l'absence d'équivalence entre le nombre de jours de prison et le montant de l'amende, il est difficile, toutefois, d'établir une règle formelle et on ne peut qu'interpréter la tendance générale qui découle des relevés effectués. À cet égard, on constate donc que le rapport amende-jours de prison est plus élevé pour la Cour des sessions de la paix que pour les Cours municipales. Cela signifie qu'il est plus «avantageux» pour un accusé d'être condamné à l'emprisonnement pour défaut de paiement d'amende, par la Cour des sessions de la paix, que par une Cour municipale.

Parallèlement, quand on divise le montant de l'amende par le nombre de jours de détention, on obtient un montant très bas 
correspondant à chaque jour purgé. Ce montant est certes plus élevé pour la Cour des sessions de la paix, mais on est encore loin d'avoir un taux qui pourrait correspondre à une certaine réalité économique. Le rapport amende-jours de prison est très arbitraire en somme, et ne semble pas refléter le rapport revenus/jours de travail, même pour les personnes considérées comme économiquement faibles.

Le véritable problème de l'emprisonnement à défaut de paiement d'amende, dans son aspect technique, ne réside donc pas dans l'imposition de l'amende elle-même, mais bien dans le rapport qui s'établit entre le nombre de jours d'emprisonnement imposé par la sentence, et le montant de l'amende. En plus de n'avoir aucune équivalence réelle entre ces deux composantes de la sentence à option, et ce, tant pour la Cour des sessions de la paix que les Cours municipales, le rapport qui existe entre l'amende et le nombre de jours de prison est généralement irréaliste comparativement au rapport revenus/jours de travail d'un individu moyen. On en arrive ainsi à des situations paradoxales comme de faire purger 8 jours à un individu qui n'a pas pu payer $\$ 24$ d'amende, ce qui représente $\$ 3$ par jour!

\section{CONCLUSIONS}

À l'époque actuelle, où prévaut la philosophie selon laquelle il convient d'utiliser la peine de prison, principalement dans les cas où elle est indispensable pour protéger la société contre les dangers d'une victimisation éventuelle, les modes de l'emprisonnement à défaut de paiement d'amende ne semblent guère conformes à cette approche. En effet, bien que le pouvoir judiciaire doit en principe s'efforcer d'éliminer les emprisonnements inutiles, et que la Commission de réforme du droit étudie les possibilités de déjudiciarisation, plus de la moitié des personnes qui ont été emprisonnées à Orsainville, au cours de l'année 1974, l'ont été pour défaut de paiement d'amende. Il est surprenant, pour ne pas dire anormal et inacceptable, que $54,3 \%$ des personnes incarcérées à Orsainville l'année dernière, l'aient été pour défaut de paiement d'amende, d'autant plus que dans $95 \%$ des cas l'amende est rattachée à des infractions de faible gravité, ne représentant en soi qu'un danger très limité pour la société.

Le phénomène de l'emprisonnement à défaut de paiement d'amende, tel qu'il existait à Orsainville, au cours de l'année 1974, 
n'est pas, en outre, un phénomène isolé. Menghile et Pépin l'avaient signalé dans leur recherche, dirigée par Pierre Landreville, sur le Centre de détention de Montréal. Ils ont trouvé, en effet, que $47,7 \%$ des détenus étaient emprisonnés pour défaut de paiement d'amende. Comment peut-on concevoir la "déjudiciarisation» en présence de telles réalités? N'y a-t-il pas lieu de se demander si à notre époque il est concevable d'emprisonner un "débiteur» de la justice à défaut de paiement de $\$ 18$ ou de $\$ 36$, ou plus... Certes on doit disposer de mesures destinées à obliger les concernés à assumer leurs responsabilités, mais ne serait-il pas préférable alors d'utiliser des alternatives telles que jours-travail social, ou jours-amende, au lieu de subsituer à ceux là les jours-prison? Voilà les questions fondamentales qu'on est en droit de se poser!

Par ailleurs dans le système actuel, il n'y a pas de relation entre les deux composantes de la sentence à option, et le plus souvent le nombre de dollars correspondant à chaque journée d'emprisonnement, est ridiculement bas. Il existe, en outre, une disproportion totale entre ce que coûte une journée d'emprisonnement et ce que rapporte une journée de travail en terme de dollars.

Pour remédier à cette situation de fait, il y aurait lieu d'instaurer dans l'immédiat, des normes administratives plus sévères. C'est ainsi qu'un juge qui déclare une personne coupable d'une infraction et décide de lui imposer une amende, devrait, pour fixer le nombre de jours d'emprisonnement pour défaut de paiement d'amende, consulter des tables d'équivalences réelles qui correspondraient à la réalité économique, des personnes comparaissant devant lui. On éviterait ainsi des disproportions telles que celles que nous avons relevées. Dès que la sentence serait rendue, il appartiendrait alors à un organisme administratif de l'appliquer, de voir à l'octroi de délais supplémentaires et de recouvrer la somme prévue par la sentence. Ce ne serait qu'en cas de refus catégorique de payer qu'on retournerait devant le juge pour que celui-ci décide d'appliquer une mesure plus sévère de jours-travail, par exemple.

On ne peut pas prétendre que dans un tel système il y aurait abdication des pouvoirs de la part du juge et il convient de souligner qu'il aurait comme avantage d'assurer l'égalité de tous devant la loi en éliminant des situations d'infériorité des économiquement faibles.

En effet, l'emprisonnement à défaut de paiement d'amende reflète les problèmes plus graves que ceux liés aux lacunes éventuel- 
les de l'organisation judiciaire. Nos centres de détention semblent être des refuges de pauvres, de personnes économiquement défavorisées et de vagabonds. Certes il s'agit là d'une réalité qui n'est guère nouvelle, ni originale, mais il apparaît urgent de rompre le cercle vicieux de certaines traditions socio-judiciaires.

À notre époque où on commence à considérer la peine privative de liberté comme une mesure extrême, on comprend mal, en somme, la nécessité de détention des personnes qui ne présentent pas de dangerosité pour la société et qui, théoriquement, pourraient être traitées de façon différente plus conforme aux principes de la défense sociale et aussi moins coûteuse pour les contribuables...

\section{BIBLIOGRAPHIE}

Description de la population de l'Établissement de détention de Montréal, par Claude Menghile et Ronald Pépin. Directeur de la recherche : Pierre Landreville, Ph.D. (rapport relié), juin 1974, 53 pages.

La Déjudiciarisation, Commission de réforme du droit du Canada. Document de travail 7, Édit. Information Canada, 1974, 30 pages.

Rapports annuels de la Direction générale de la probation et des établissements de détention de la province de Québec. 1973-1974. (document polycopié).

HUDON, M. (1974) : Recherche sur la population du Centre de détention de Montréal. École de criminologie, Université de Montréal. 